\title{
Helmut Rösing, Albrecht Schneider, Martin Pfleiderer (Hrsg.): Musikwissenschaft und populäre Musik. Versuch einer Bestandsaufnahme (Hamburger Jahrbuch für Musikwissenschaft 19), Frankfurt/M.: Peter Lang, 2002.
}

\author{
Rezension von Ralf von Appen
}

$-1-$

\begin{abstract}
"Geht man davon aus, dass es Aufgabe von Musikwissenschaft ist, sich mit allen musikalischen Erscheinungsformen in Vergangenheit und Gegenwart zu befassen, dann müsste die wissenschaftliche Auseinandersetzung mit populärer Musik, kurz, die Popularmusikforschung im 20. Jahrhundert ein gewichtiger Gegenstandsbereich dieser Disziplin sein",
\end{abstract}

so Helmut Rösing nüchtern am Anfang seines Beitrags zur Geschichte der Popularmusikforschung in der Bundesrepublik Deutschland, die diesen Sammelband eröffnet (S. 13). Doch ganz offensichtlich bildet die Beschäftigung mit Jazz, Schlager, Volkssowie Pop- und Rockmusik auch gut 40 Jahre nach den ersten diesbezüglichen Studien immer noch den Sonderfall an musikwissenschaftlichen Instituten und in den zentralen Publikationsorganen. So befassen sich beispielsweise im Wintersemester 2003/2004 weniger als drei Prozent der gut 1000 an die Musikforschung gemeldeten Veranstaltungen in der BRD wissenschaftlich-theoretisch mit Pop- oder Rockmusik. Der Anteil Pop-relevanter Artikel im zehnten Band der neuen $M G G$ (Personenteil) beträgt 0,5 Prozent, weitere 0,5 Prozent befassen sich mit Jazz-Musikern. In der Musikforschung ist bislang exakt ein Aufsatz zum Thema erschienen, genau einer auch in der Zeitschrift Musik \& Ästhetik. Popularmusikforschung ist nur an sehr wenigen Hochschulen verankert, die Anzahl der bundesdeutschen Universitäten mit mehr als zwei theoretischen Veranstaltungen pro Semester beträgt: zwei. Zum Vergleich: Der Anteil von KlassikAufnahmen an allen verkauften Longplay-CDs war im Jahr 2002 mit 7,2 Prozent der niedrigste in den letzten zehn Jahren. ${ }^{1}$

Jahreswirtschaftsbericht 2002 des Bundesverbandes der Phonographischen Wirtschaft e.V., http://www.ifpi.de/jb/2003/48-53.pdf, S. 51. 
Zwischen den Diskursen der Popularmusikwissenschaft und der traditionellen Musikforschung verläuft leider immer noch ein Graben. Die überwiegend historisch orientierte Musikwissenschaft nimmt die Arbeit der Popforscher nur sehr selten zur Kenntnis, diese wiederum schütten häufig gleichsam das Kind mit dem Bad aus und halten die Methoden und Themen der bisherigen Musikwissenschaft für überkommen und irrelevant, nach dem Prinzip: "Was die alles falsch gemacht haben, wollen wir nicht wiederholen." Konstruktive Zusammenarbeit und gegenseitige Anregung finden viel zu selten statt. ${ }^{2}$

\section{$-3-$}

Umso bemerkenswerter ist es, dass die vorliegende 19. Ausgabe des bislang der so genannten Kunstmusik vorbehaltenen Hamburger Jahrbuchs für Musikwissenschaft diese Grenze überschreitet. Das Musikwissenschaftliche Institut der Universität Hamburg, das diese Reihe herausgibt, ist eines der wenigen mit einem Schwerpunkt auf der Systematischen Musikwissenschaft und als solches sehr um Offenheit gegenüber dem musikalischen Material bemüht. Zudem fühlt man sich dem Anspruch verpflichtet, vor allem Musikformen mit besonderer gesellschaftlicher Relevanz zu untersuchen, um Musikwissenschaft sinnvoll als Kulturwissenschaft betreiben zu können.

\section{$-4-$}

Der vorliegende Band Musikwissenschaft und populäre Musik. Versuch einer Bestandsaufnahme ist in vier Sektionen gegliedert. Im ersten Block geht es um die Forschungsgeschichte und die Fragen, die das interdisziplinäre Projekt 'Popforschung' im Kern zusammenhalten. Dazu steuert Helmut Rösing eine konzise und äußerst informative Darstellung wesentlicher Entwicklungen und Veröffentlichungen der deutschen Popularmusikforschung bei, während Martin Pfleiderer das gleiche für den Bereich des Jazz unternimmt und Paul Riggenbach speziell Publikationen mit empirischer Ausrichtung fokussiert. Essentielle Grundsatzfragen nach Sinn, Zielen, Erkenntnisinteressen, Adressaten und Methoden stellen neben Rösing vor allem Peter Wicke (Popmusik in der Theorie. Aspekte einer problematischen Beziehung) und Dietrich Helms (Musikwissenschaftliche Analyse populärer Musik?). Diesen beiden Arbeiten kommt aufgrund ihrer

2 Eine positive Ausnahme bildet z.B. Ulrich Tadday (2004): "Musikalische Körper - körperliche Musik: Zur Ästhetik auch der populären Musik". In: Handbuch der Musikästhetik. Hg. v. Helga de la Motte-Haber (=Handbuch der Systematischen Musikwissenschaft 1). Laaber: Laaber, S. 395-407. Dort heißt es: "Wurde die Ästhetik der populären Musik in der Vergangenheit durch die Ästhetik der nicht-populären Musik deformiert, wird die Ästhetik der nicht-populären Musik in Zukunft durch die Ästhetik der populären Musik reformiert" (S. 395). 
Allgemeinheit und der Tragweite ihrer Thesen die größte Bedeutung innerhalb des Buches zu, sie laden daher zu genauerer Auseinandersetzung ein:

$-5-$

Wicke bezweifelt in seinen Reflexionen zunächst provokativ, wenngleich mit bedenkenswerten Argumenten die Legitimität akademischer Popforschung, um sie dann als zentrale Teildisziplin kritischer Kulturwissenschaft zu re-etablieren. Zu dieser Aufwertung kommt er über eine Neudefinition des Musikbegriffes, bei dem der gemeinhin als zentral angesehene klangliche Aspekt zugunsten des sozialen in den Hintergrund tritt. Demzufolge soll es nach Wicke in der (Popular-)Musikforschung nicht um die M u s i k, um Werke und Strukturen von Klangereignissen gehen (schon gar nicht um den "[skurrilen Versuch], die Pop-Erfahrung in der Begrifflichkeit von Kunsttheorie zu fassen", S. 61), sondern um M u s i a ls M e di u m gesellschaftlicher Prozesse und (Macht-)Verhältnisse - ein nicht nur für den populären Bereich durchaus vielversprechender Ansatz, der sich allerdings dem Problem stellen muss, dass das im engeren Sinn 'Hörbare' aus den Erklärungen allzu schnell weggekürzt wird oder zur Blackbox verkommt. Dieses Problem sieht auch Wicke, und in einem den Beitrag von Rösing sehr gut ergänzenden internationalen Forschungsüberblick zeigt er mit scharfsinniger Kritik die diesbezüglichen Fehler bisheriger Ansätze zur Erforschung populärer Musik auf. Um Popmusik nun angemessen erfassen zu können, müsse die Musikwissenschaft Wicke zufolge zwei ihrer Grundansichten radikal ändern: Zum einen müsse erkannt werden, dass die tradierte, selbstverständlich scheinende Reduktion des Begriffes Musik auf die Dimension des Klanglichen eine problematische Konstruktion sei. Gerade im Bereich der Popmusik sei die Dominanz des Klanglichen sogar in Zweifel zu ziehen. Vielmehr versteht Wicke Musik als "ein soziales Faktum [...], das sich in der Materialität des Mediums Klang vermittelt" (S. 70). Zu fragen sei immer von neuem, "wie Begriff und Praxis von Musik sozial konstituiert sind" (ebd.), "auf welche je besondere Weise und nach welchen Regeln sowie in welchen diskursiven und technologischen Vermittlungszusammenhängen Klang hier jeweils als Musik funktioniert" (S. 69). Zum anderen müsse man aufhören, Musik immer nur auf einen in ihr versteckten, semantisch zu deutenden 'Inhalt' hin zu analysieren. Bei diesem beschränkten Verständnis von Kultur als Kommunikation würden zentrale Momente der Popmusik - nämlich Sinnlichkeit, Vergnügen und Unterhaltung, Spiel, Tanz und Körpererfahrung - übersehen werden. Führte man diese beiden Paradigmenwechsel in der Musikwissenschaft konsequent durch, dann könnte sich, so Wicke, die Popularmusikforschung zu einer zentralen kulturwissenschaftlichen Disziplin entwickeln: 
"Letztlich also geht es um nicht weniger als darum, die populären Musikformen für eine Analyse aufzuschließen, die Einsicht in das Innenleben der Mediengesellschaft und der sie konstituierenden Machtverhältnisse, in die darin aufgehobenen Hoffnungen, Wünsche, Sehnsüchte, Phantasien und Triebstrukturen gewährt. Dass solche Einsichten nur in Auseinandersetzung mit den populären Kulturformen zu gewinnen sind, weil nur hier sich diese Seite des gesellschaftlichen Lebens offenbart, macht auch die theoretische Analyse populärer Musik zu einem unverzichtbaren Bestandteil einer jeden kritischen Kultur- und Gesellschaftstheorie, die sich auf der Höhe ihrer Zeit befinden will" (S. 62).

Ein solcher Anspruch lässt gespannt auf umfassende Durchführungen dieser Gedanken am konkreten Beispiel warten. Erst in der Ausführung kann sich zeigen, ob sich der erhoffte Einblick in die Gesellschaft und ihre Kultur plausibel mit dem klanglich Gestalteten in Einklang bringen lässt.

$-6-$

Als zentrale musikwissenschaftliche Kompetenz gilt die Analyse. Doch wie kann eine Analyse für den Forscher aussehen, dem es einerseits um weit mehr als das eigentliche musikalische Material geht, der aber andererseits auch die musikalische Gestalt ernst nehmen will? Dietrich Helms argumentiert gegen hermeneutische, rein formale oder sonstige aus der Kunstmusik abgeleitete, werkimmanente Verfahren und sieht allein in der Analyse der Rezeption ein relevantes Forschungsfeld, von dem auch Akzeptanz im Pop-Diskurs selbst zu erwarten sei: "Bedeutungen - einschließlich aller Funktionalisierungen - werden an die Musik herangetragen, nicht aus ihr herausinterpretiert. [...] Eine Analyse populärer Musik im Kontext des Diskurses der populären Musik muss daher eine Analyse des Rezeptionskontextes, $d . h$. der Prozesse, die einem Stück seine Bedeutung zuweisen, sein" (S. 102). Die schwer einzugrenzende Grundlage der Analyse sollten somit nicht nur Aufnahmen (schon gar nicht Notentexte) sein, sondern die möglichen Zeichenfunktionen von Videoclips, Frisuren, Images oder Sounds sowie das soziale, psychische und historische Umfeld des Hörers oder - interessanter - bestimmter Rezipientengruppen. Ob die jeweils vorgenommenen Zuschreibungen durch das Stück legitimiert, also 'wahr' oder 'falsch' sind, ist aus dieser Perspektive gleichgültig und, so Helms, auch gar nicht zu entscheiden, denn: 
"Die Parameter Melodik, Harmonik und Rhythmik lösen keine soziale Gruppenbildung aus. Ihnen kann durch soziale Prozesse zwar eine bestimmte Zeichenfunktion zugeordnet werden, die wiederum durch soziale Prozesse gelernt und wieder erkannt wird. Dieser Vorgang ist jedoch nicht abhängig von der Tonfolge, sondern nur von früheren sozialen Prozessen, die einer ähnlichen Tonfolge bereits Bedeutungen zugewiesen haben" (S. 96).

Dem ist im Großen und Ganzen zuzustimmen. Aber es ist bedauerlich, dass mit dieser ausschließlichen Betonung des Sozialen und damit der Austauschbarkeit solcher Zuschreibungen auf die überaus interessante Frage nach dem möglicherweise doch manchmal in der musikalischen Struktur angelegten Potential von Anschlussmöglichkeiten verzichtet wird. Spricht nicht ein Ska-Rhythmus den Körper anders an als Radiohead'sche Polyrhythmik? Wird dem 'dreckigen' Lo-Fi Sound der White Stripes rein willkürlich eine andere Bedeutung zugeschrieben als den sich technisch auf dem Stand der Zeit befindlichen Produktionen eines Dieter Bohlen? Ist es nur durch soziale Prozesse zu begründen, dass die Terz-orientierte Beatles-Harmonik andere Menschen anspricht, andere psychische Funktionen erfüllt und kommerziell erfolgreicher ist als die von kleinen Sekunden und Tritoni geprägten Stücke Metallicas? Gerade die Zusammenführung traditioneller Analyse-Methoden mit dem neuen Blick auf soziale, psychische und historische Rezeptionskontexte wäre doch sinnvoll, um die Rezeption (und auch die Produktion) von Musik zu verstehen, ohne die Musik dabei zu vernachlässigen. Wie bei Wicke bildet also auch hier wieder der Musikbegriff das Zentrum des Problems. Dementsprechend schließt Helms, dass eine aus dem Popdiskurs (und nicht aus der institutionalisierten Musikologie) heraus entstehende Wissenschaft "nicht zwangsläufig eine Musik-Wissenschaft" sein müsse (S. 101).

\section{$-7-$}

Detaillierteren Fragen, die im weiteren Sinn um das Problem der musikalischen Analyse kreisen, widmet sich dann der zweite Block. Hier stellt Albrecht Schneider objektive Messverfahren als neue Methoden der Klanganalyse vor, von denen er sich Ergebnisse über ungewöhnliche Spektren, über Sachverhalte der Wahrnehmung komplexer Klangfolgen, über den Entstehungsprozess von Aufnahmen sowie wertvolle Dienste bei der Transkription erhofft: konventionelle Probleme der Musikwissenschaft, für die Wicke und Helms vermutlich kein Interesse aufbringen würden. Werner Jauck arbeitet in seinem Beitrag die hohe Bedeutung der Körperlichkeit für die Popmusik heraus. Er sieht 
dabei den Sound "wegen seiner inhaltlichen Unbestimmtheit, zugleich ob seiner unmittelbar emotionalen Wirkung" (S. 134) als den zentralen Parameter an. Anders als Helms geht es ihm um deutliche strukturelle Bezüge zwischen der Musik und ihrer Aufnahme durch den Hörer, die vor allem über den körperlich unmittelbar erregenden Sound funktionieren, nicht über leicht misszuverstehende Codierungen: "Klang und körperliches Verhalten sind Teile eines emotionalen Zustandes und nicht ihr zeichenhafter Ausdruck" (S. 135). Weitere Aufsätze dieses Abschnittes widmen sich der Analyse von Filmmusik oder der Verschmelzung von Folk und Rock, der kulturwissenschaftlichen Interpretation von Transformationsprozessen lokaler Musikpraktiken auf dem globalen Markt sowie dem musikalischen Distinktionsverhalten von Schülern in multikulturellen Klassen.

$-8-$

Seiner Hamburger Herkunft trägt der Band im dritten Abschnitt Rechnung: Hier werden konkrete Fragen des Hamburger Musikgeschehens behandelt, die zum Teil als exemplarisch angesehen werden können und somit auch durchaus für nicht Ortsansässige interessant sind. So zeichnet Bernd Hoffmann anhand alter Clubzeitschriften die Entwicklung der lokalen Hamburger Jazz-Szene der 1950er Jahre nach. Andere Autorinnen und Autoren beleuchten den enormen Erfolg Hamburgs als Musical-Standort oder untersuchen die dortigen Lebens- und Arbeitsbedingungen einiger Jazzmusikerinnen. Roger Behrens analysiert die jüngere Popgeschichte der Stadt und argumentiert gegen die Verwendung des Labels 'Hamburger Schule' für Bands wie Blumfeld, Tocotronic oder Die Sterne.

$-9-$

Der vierte Block schließlich ist mit dem Schlagwort "Bewertungsfragen" überschrieben. Leider fehlt hier eine den Arbeiten von Rösing, Helms und Wicke adäquate Einführung in Fragen der Ästhetik populärer Musik. Stattdessen finden sich Partikularuntersuchungen, die sich teilweise widersprechen. Dietmar Schlumbohm sieht aus einer viel zu stark musikindustriell geprägten Perspektive in der zunehmenden Verbreitung digitaler Medien eine gefährliche Wertminderung der Musik. Günther Jacob untersucht die typischen Distinktionsprozesse, in denen der Popdiskurs über 'In' und 'Out' entscheidet, und wer dabei über die Definitionsmacht verfügt. Martin Büsser bemüht sich um eine Versöhnung einzelner Bereiche der Popmusik mit Adorno, indem er den Blick auf einige Avantgardisten der Popmusik lenkt, die weder ein Massenpublikum bedienen wollten noch sich "materialästhetisch von avantgardistischen Experimenten in der bildenden Kunst und Literatur" (S. 321) unterschieden. Dementsprechend präsentiert 
Büsser eine distinktionsträchtige "'geheime' Popgeschichte jenseits der Warenlogik" (S. 322). Dabei spielt er ästhetischen Gehalt (die Guten) und kommerzielle Orientierung (die Bösen) gegeneinander aus. Abgesehen davon, dass es für ästhetische Qualität keine objektiven, d.h. allgemeingültigen Kriterien geben kann, ist eine solche Trennung weder sinnvoll (man denke an die hier nicht einzuordnenden Sex Pistols oder die Pet Shop Boys) noch wissenschaftlich erwünscht: Will man Musikwissenschaft als Kulturwissenschaft betreiben, wofür Rösing und Wicke in ihren Beiträgen ja überzeugend plädieren, dann sollte man auf subjektive Wertungen zumindest im wissenschaftlichen Kontext verzichten und nicht die alte, elitäre Grenze zwischen U und E innerhalb der Popmusik neu ziehen.

$$
-10-
$$

Alles in allem stellt der Band eine sehr wichtige Veröffentlichung dar, dem der Wert eines Standardwerkes (wie etwa den von Helmut Rösing mitherausgegebenen Handbüchern zu Musikpsychologie und Musikwissenschaft) zugesprochen werden könnte, enthielte er nicht auch einige thematisch unbedeutende Aufsätze von geringerem allgemeinen Interesse. Aber auch in dieser Form bietet die "Bestandsaufnahme" eine Standortbestimmung der Popularmusikforschung, die im deutschsprachigen Raum ohne Beispiel ist und auch international neben z.B. Popular Music Studies von David Hesmondhalgh und Keith Negus (London 2002) oder Rock over the edge von Roger Beebe, Denise Fulbrook und Ben Saunders (Durham 2002) bestehen kann. Es wäre schön, wenn dieser Band viele Leserinnen und Leser finden würde: aus dem Bereich der traditionellen Musikwissenschaft, die sich hier ein Bild von den zahlreichen Forschungsgebieten ihrer Kolleginnen und Kollegen machen und so manches Vorurteil abbauen oder einen neuen Blick auf das eigene Forschungsfeld gewinnen könnten; und aus dem Bereich der Studierenden: so mancher Funke könnte hier gezündet werden. Doch leider wird das Erreichen der studentischen Leserschaft durch einen Preis von 56 Euro nicht eben leicht gemacht. 